\title{
Über den Ursprung der Magnetfelder auf Sternen und im interstellaren Raum
}

\author{
Von Ludwig BiermanN \\ Mit einem Anhang von A.Schlüter \\ Aus dem Max-Planck-Institut für Physik, Göttingen \\ (Z. Naturforschg. 5a, 65-71 [1950]; eingegangen am 20. Juni 1949)
}

\begin{abstract}
Es wird gezeigt, daß im Falle stationärer wirbelnder nicht-massenproportionaler Kräfte in einem Plasma elektrische Ströme fließen müssen, und daß die so entstehenden elektrischen Ströme im Sterninnern Magnetfelder erheblicher Stärke erzeugen. Die bei hydrostatischem Gleichgewicht hierdurch geforderte Neigung der isobaren gegen die isothermen Flächen entsteht z. B., wenn die gesamte, den Druckgradienten bestimmende Beschleunigung (Schwerkraft und Rotation bzw. Wirbel) einen potentiallosen Anteil $g^{*}$ besitzt. Es ergibt sich, daß im Sterninnern Beträge von $g^{*}$ der Ordnung $1 \mathrm{~cm} / \mathrm{sec}^{2}$ schon Magnetfelder von $10^{3}$ Gauß hervorrufen. Der einzige bisher analytisch durchdiskutierte Fall (barokline Rotation des ganzen Sterns) bestätigt die gezogenen Schlüsse, führt aber nicht zu einer Deutung der Gestalt der auf der Sonne und auf einigen vermutlich rasch rotierenden Sternen festgestellten Magnetfelder. Es werden daher qualitativ zwei weitere Möglichkeiten diskutiert. Führt man die Sonnenflecken mit Bjerknes auf geschlossene hydrodynamische Wirbelringe zurück, so führen diese wegen der Störung des Feldes der Rotationsgeschwindigkeit zu $[\nabla P \nabla T] \neq 0$; die entstehenden elektrischen Ströme ergeben Magnetfelder parallel zur Wirbelachse, die in den beiden Wirbelringen jeder Hemisphäre antiparallel sind. Für rasch rotierende Sterne andererseits wird ein Modell vorgeschlagen, das durch die in irdischen Drehstürmen beobachteten Verhältnisse nahegelegt wird. Es werden verhältnismäßig rasche meridionale Zirkulationen vorausgesetzt, welche auf Beschleunigungskomponenten um die Rotationsachse herum führen; diese ergeben axiale Magnetfelder. Zum Schluß wird die Anwendung dieser Betrachtungen auf die Entstehung interstellarer Magnetfelder kurz skizziert.

Im Anhang wird ein spezielles Modell eines nicht starr rotierenden Sterns durchgerechnet; die entstehenden Magnetfelder werden angegeben.
\end{abstract}

$\mathrm{D}$ ie Entdeckung von Magnetfeldern auf einer Anzahl vermutlich rasch rotierender Sterne und die große Bedeutung interstellarer elektromagnetischer Felder für die Theorie der Ultrastrahlung haben die Frage nach dem Ursprung dieser Felder wieder in den Vordergrund gerückt. Es sollen daher einige Überlegungen mitgeteilt werden, welche in allgemeiner Weise dartun, wie man auf der Grundlage der kinetischen Gastheorie und der Elektronentheorie die Existenz starker Magnetfelder auf rotierenden Sternen verstehen kann, und welche Felder im interstellaren Raum zu erwarten sind ${ }^{1}$.

1 Ein großer Teil der in dieser Note beschriebenen Überlegungen ist in den Jahren 1939-1945 entstanden und in einem unveröff. gebliebenen Bericht zusammengefaßt worden. - Für die Deutung des Magnetfeldes der Erde hat Els a s e r thermoelektrische Ströme im Erdkern vorgeschlagen (Physic. Rev. 55, 489 [1939], 69, 106 [1946], 70, 202 [1946], 72, 821 [1947]). — Vgl. ferner T. G. C ow ling, Monthly Notices Roy. astronom. Soc. 105, 166 [1945] sowie H. Alfvén, Ark. Mat. Astronom. Fysik, Ser. B 29, Nr. 2 [1942]; ibid. Ser. A, Nr. 12 [1943]; C. W a lé n, ibid. Ser. A 30, Nr. 15 [1944].
1. Wir gehen aus von der Gleichung für die Geschwindigkeit $\mathfrak{D}=\mathfrak{b}_{\mathrm{i}}-\mathfrak{v}_{\mathrm{e}}$ der Diffusion des Elektronengases durch das Ionengas hindurch, wo $\mathfrak{v}_{i}$ und $\mathfrak{v}_{\mathrm{e}}$ die makroskopischen Geschwindigkeiten der beiden Komponenten sind, und betrachten sofort den Fall unmerklicher Entmischung $N_{\mathrm{i}}=N_{\mathrm{e}}=N\left[\mathrm{~cm}^{-3}\right]$ (keine Ladungstrennung). Die Beschleunigungen $\mathfrak{F}_{\text {e }}$ und $\mathfrak{F}_{\mathrm{p}}\left[\mathrm{cm} / \mathrm{sec}^{2}\right]$ seien durch auf die einzelnen Teilchen wirkende äußere Kräfte (elektrische Felder, Strahlungsdruck) hervorgerufen. $p_{\mathrm{i}}+p_{\mathrm{e}}=p_{\mathrm{G}}$ $=2 N k T$ sei der Gasdruck. Dann gilt ${ }^{2}$

$$
\begin{array}{r}
\delta=-2 D_{12}\left\{\frac{m_{\mathrm{p}}-m_{\mathrm{e}}}{m_{\mathrm{p}}+m_{\mathrm{e}}} \nabla \log p_{\mathrm{G}}-\frac{m_{\mathrm{e}}}{k T^{\prime}} \frac{m_{\mathrm{i}}}{m_{\mathrm{i}}+m_{\mathrm{e}}}\right. \\
\left.\left(\mathfrak{F}_{\mathrm{e}}-\mathfrak{F}_{\mathrm{i}}\right)\right\}+D_{T} \frac{\partial \ln T}{\partial \mathfrak{r}}\left[\frac{\mathrm{cm}}{\mathrm{sec}}\right]
\end{array}
$$

2 J e a ns, Dynamical Theory of Gases, Cambridge 1925, Ch. XIII; C h a p m a n - C ow ling, Mathematical Theory of nonuniform Gases [1939]. 
$=\frac{2 D_{12}}{l \cdot T}\left\{-\frac{1}{2 N} \nabla p_{\mathrm{G}}+m_{\mathrm{e}}\left(\widetilde{F}_{\mathrm{e}}-\mathfrak{F}_{\mathrm{i}}\right)\right\}+I_{T} \frac{\partial \ln T}{\partial \mathfrak{x}}$,

worin jetzt $m_{\mathrm{p}} \pm m_{\mathrm{e}}=m_{\mathrm{p}}$ gesetzt ist. Der in $\widetilde{F}$ enthaltene Strahlungsdruck wirkt nur auf die Elektronen, und zwar um den Faktor $f$ (dimensionslos) stärker als nach der klassischen Theorie ${ }^{3}$. Auch ohne nichtmassen-proportionale äußere Kräfte $m \mathfrak{F}$ würde sich ein elektrischer Strom der Dichte i (e ElementarLadung in el. st. E.

$$
\left.\mathrm{j}=+e \mathrm{ND} \text { [el. st. E. } / \mathrm{cm}^{2} \mathrm{sec}\right]
$$

ergeben, der dadurch zustande kommt, daß die Druckgradienten im wesentlichen dieselbe Kraft auf Protonen und Elektronen ausüben, so daß die Beschleunigungen im reziproken Verhältnis der Massen verschieden sind.

Die Druckgradienten und die andern diffusionserzeugenden Kräfte gemäß Gl.(1) stellen also eine eingeprägte elektromotorische Kraft dar gemäß

$$
e \mathfrak{E}^{\mathrm{e}}=\frac{1}{2 N} \nabla p_{\mathrm{G}}-\frac{1}{2} \frac{D_{T}}{D_{12}} k \nabla T,
$$

wo jetzt die Strahlungskraft vernachlässigt ist.

Hat $(1 / N) \nabla p_{\mathrm{G}}$ ein Potential, wie im Falle der Schwere und (z. B.) starrer Rotation, so fallen die Flächen gleichen Drucks und gleicher Temperatur zusammen; daher hat auch die Strahlungskraft ein Potential, und es wird ein stromloser Zustand erreicht durch ein elektrostatisches Feld

$$
-e \mathfrak{E}=\frac{1}{2 N} \nabla l_{\mathrm{S}}-\frac{1}{2} \frac{D_{T}}{D_{12}} k \nabla T,
$$

das sich automatisch durch eine äußerst geringe Trennung der Ladungen einstellt.

Hat nun aber das Vektorfeld ${ }^{\mathrm{e}}$ e auch einen Anteil, dessen Rotation nicht verschwindet, so ist für diesen Anteil offenbar keine Kompensation durch ein elektrostatisches Feld möglich. Es müssen Ströme fließen, welche Magnetfelder erzeugen. Es gilt im stationären Fall

$$
\operatorname{div} \mathbf{j}=\operatorname{div}\left(\sigma\left[\mathfrak{E}^{\mathrm{e}}+\mathfrak{E}\right]\right)=0,
$$

$$
\text { mit } \operatorname{rot} \mathfrak{E}=0 \text {, }
$$

wo jetzt gemäß $\operatorname{rot} \mathfrak{S}=\frac{4 \pi}{c} \mathrm{i}$,

$$
\sigma=2 \frac{e^{2}}{k T} D_{1}
$$

statt $D_{12}$ die Leitfähigkeit $\sigma\left[\mathrm{sec}^{-1}\right]$ eingeführt ist.

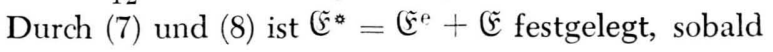

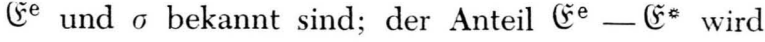
ganz durch Raumladungen kompensiert, $\tilde{E}^{*}$ nur im Falle $\nabla \sigma \neq 0$ zum Teil.

2. Wir zeigen zunächst, daß im tiefen Innern eines Sterns der Hauptreihe des Russell-Diagramms, wo $\sigma$ zu $10^{17}$ angenommen werden darf ${ }^{4}$, winzige Beträge von $\mathfrak{E}^{*}$ schon große magnetische Feldstärken ergeben. Definiert man eine Beschleunigung $\mathfrak{g}^{*}$ durch

$$
e\left(\mathfrak{r}^{*}=m g^{*} . \quad\left(m=\frac{m_{\mathrm{i}}+m_{\mathrm{e}}}{2} \approx \frac{m_{\mathrm{i}}}{2}\right)\right.
$$

und setzt $\mathfrak{g}^{*}=1 \mathrm{~cm} / \mathrm{sec}^{2}$, so folgt $\mathfrak{r}^{*} \approx 10^{-15}$ el. st. E. und für rot $\approx 10^{-10,5} \mathrm{~cm}^{-1}$

$$
I I \approx 10^{3} .
$$

Fragt man nun, unter welchen Bedingungen Wirbelbeschleunigungen $\mathfrak{g}^{*}$ stationär vorhanden sein können, so zeigt sich als nächstliegende die Möglichkeit stationärer reiner Rotation mit $\partial_{\omega} / \partial_{z} \neq 0$, wo $\omega(z, \bar{r})$ [ $\left.\mathrm{sec}^{-1}\right]$ die Winkelgeschwindigkeit der Rotation ist, die $z$-Achse die Rotationsachse und $\vec{r}$ der Abstand von ihr. In diesem Fall ist die Rotation der Zentrifugalbeschleunigung $\neq 0$, während im anderen Fall $\left(\partial_{\omega} / \partial z\right.$ $=0, \partial \omega / \partial \vec{r}$ beliebig) die Zentrifugalbeschleunigung ein Potential besitzt. Definiert man eine Geschwindigkeit $v_{\mathrm{r}}^{*}$ durch

$$
g^{*}=\frac{\left(v_{r}^{*}\right)^{2}}{r},
$$

so erkennt man, welche Geschwindigkeitsbeträge $v_{\mathrm{r}}{ }^{*}$ erforderlich sind. Setzt man $\operatorname{rot} \approx 1 / r$, so folgt

$$
H \approx \frac{4 \pi}{e c} \sigma \bar{m}\left(v_{r}^{*}\right)^{2}=10^{+3}\left(\frac{\sigma}{10^{17}}\right)\left(\frac{v_{r}^{*}}{10^{5}}\right)^{2},
$$

worin $4 \pi / e c$ numerisch $=10^{-0,1}$ ist.

Die Wechselwirkung des Magnetfeldes mit der Sternmaterie ergibt die Kraftdichte

$$
\begin{aligned}
l_{i} & =\frac{1}{c}[\mathrm{j} \mathfrak{S}]=\frac{-1}{4 \pi}[\mathfrak{S} \operatorname{rot} \mathfrak{S}] \\
& =\frac{-1}{8 \pi} \nabla \mathfrak{S}^{2}+\frac{1}{4 \pi}(\mathfrak{S} \text { grad }) \mathfrak{S} .
\end{aligned}
$$

${ }^{3}$ A. S o m merfeld, Atombau und Spektrallinien, Bd. II, Kap. 6. Die Strahlungskraft pro Elektron beträgt im einfachsten Fall (Energietransport durch Strahlung) daher $\left(f / N_{\mathrm{e}}\right) \nabla p_{\mathrm{R}}$, wo $p_{\mathrm{R}}$ der Strahlungsdruck ist. Der Faktor $f$ hat für den Photoeffekt aus der K-Schale eines Atoms mit $h \bar{v} \gg \%$, wo $\%$ die Bindungsenergie des Elektrons ist, den Wert 8/5; im allgemeinen liegt er zwischen $8 / 5$ und 1 .

${ }_{4}^{4}$ Dieser Wert entspricht einer Temperatur von einigen Millionen Grad. 
Zerlegt man $k$ in einen wirbelfreien Anteil $k_{1}$ und einen quellenfreien Anteil $k_{2}$, so gilt

$$
\operatorname{rot} k_{1}=0, \quad 4 \pi \operatorname{div} l_{i_{1}}=-(\mathfrak{S} \Delta \mathfrak{S})-(\operatorname{rot} \mathfrak{S})^{2},
$$

$$
4 \pi \operatorname{rot} k_{2}=\operatorname{rot}(\mathfrak{S} \operatorname{grad}) \mathfrak{S} \text {. }
$$

$k$ liefert also einen Beitrag zur Druckverteilung, wobei $k_{2}$ im stationären Fall allerdings durch zirkulationserregende Beschleunigungsfelder kompensiert werden muß.

Im Fall der Zylindersymmetrie (Koordinaten $\boldsymbol{r}, \varphi$, z) und (z. B.) der Existenz nur einer $\varphi$-Komponente von $\mathfrak{g}$, ist die vollständig wirbelfreie Komponente $(-1 / 8 \pi) \Delta \mathfrak{S C}^{2}$ überall $\perp \varphi$, während die andere Komponente überall $\| \bar{r}$ und vom Betrage $\mathfrak{g}^{2} / 4 \pi \bar{r}$ ist. Die letztere hat einen wirbelnden Anteil, der aber im tiefen Innern der Sterne im allgemeinen klein gegen $\varrho g^{*}$ ist. Es gilt

$$
\begin{aligned}
\frac{1}{4 \pi} \frac{\mathfrak{Q}^{2} / \bar{r}}{\varrho g^{*}} & \approx \frac{4 \cdot \pi}{e^{2}}\left(\frac{\sigma^{2}}{\varrho}\right) \bar{m}^{2}\left(\frac{v^{*}}{c}\right)^{2} \\
& =10^{-5} \frac{1}{\varrho}\left(\frac{\sigma}{10^{1 \mathrm{i}}}\right)^{2}\left(\frac{v^{*}}{10^{5}}\right)^{2} .
\end{aligned}
$$

Darin ist $\sigma^{2} / Q-p_{\mathrm{R}} / p_{\mathrm{G}}$.

Im allgemeinsten Fall stationärer Bewegungen bei hydrostatischem Gleichgewicht lautet die hydrodynamische Bewegungsgleichung in der Eulerschen Form bei Vernachlässigung der Reibung ( $\Phi$ Schwerepotential)

$$
(\mathfrak{v} \operatorname{grad}) \mathfrak{v}=\nabla \frac{\mathfrak{v}^{2}}{2}-[\mathfrak{v} \operatorname{rot} \mathfrak{v}]=-\nabla \omega-\frac{1}{\varrho} \nabla P .
$$

Die Bedingung für das Vorhandensein von Wirbelströmen ergibt also für das Geschwindigkeitsfeld die Beziehung

$\operatorname{rot}\left(\frac{1}{\varrho} \nabla P\right)=\left[\nabla \frac{1}{\varrho} \nabla P\right]=\operatorname{rot}[\mathfrak{v} \operatorname{rot} \mathfrak{v}] \neq 0$.

Diese allgemeinere Form ist von Interesse, wenn sich der Rotation meridionale Zirkulationen oder stationäre Wirbel überlagern, bei denen die Zustandsänderungen nicht adiabatisch sind. Längs mitbewegter „,lüssiger“ Linien, auf denen die Zustandsänderungen adiabatisch sind, ist

$$
\oint \frac{1}{\varrho} d P=\iint\left[\nabla \frac{1}{\varrho} \nabla P\right] d F=0 .
$$

$5 \mathrm{Zu}$ den folgenden Ausführungen vgl. Biermann, Z. Astrophysik 25, 135 [1948].
3. Wir wollen jetzt die allgemeinen Betrachtungen der Abschn. 1 und 2 auf das Sterninnere anwenden. Die Theorie des inneren Aufbaus zeigt, daß der (konvektive) Energietransport in ausgedehnten Konvektionszonen (z. B. bestimmt in der Mittelpunktskonvektionszone, in welcher der Kohlenstoffzyklus läuft) $\perp z$ durch die Rotation behindert wird, $\| z$ dagegen nicht ${ }^{5}$. Hierdurch entstehen langsame (nichtadiabatische) meridionale Zirkulationen, welche dann aber außer der Wärmeenergie auch Rotationsmoment transportieren und hierdurch eine Abweichung des Rotationsgesetzes vom Zustand starrer Rotation erzwingen mit (im allgemeinen Fall) $\frac{\partial(\rho)}{\partial z} \neq 0$. Nach dem Satz von Bjerknes, der aus Gl. (18) hervorgeht,

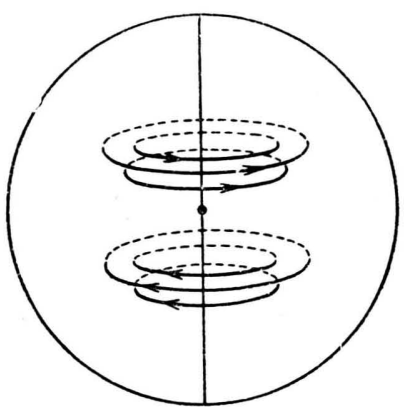

Abb. 1. Modellverteilung der magnetischen Feldstärke. Vorausgesetzt ist schnellere Rotation der mittelpunktsnahen Zonen des Sterns.

wenn man für $\mathfrak{v}$ die Rotationsgeschwindigkeit einsetzt, führt dies zu einer stationären Neigung der isothermen gegen die isobaren Flächen, für deren Größenordnung sich $\frac{r_{r}{ }^{2}}{\bar{r} g}$ ergibt; dabei sorgen die meridionalen Zirkulationen für die Aufrechterhaltung des richtigen Rotationszustandes und des Wärmegleichgewichts. Daher wird in Sternen mit subatomaren Energiequellen der Fall der Rotation mit $\partial \omega / \partial z \neq 0$ der allgemein realisierte sein. Auch die Beobachtungen des Rotationsgesetzes der Sonnenoberfläche und der langlebigen Fleckenherde deutete auf diesen Typus der Rotation. Das spezielle, freilich ohne Berücksichtigung der Zirkulationen berechnete Modell der Sonnenrotation von M. Schwarzschild ${ }^{5 a}$ zeigt ebenfalls $\partial \omega / \partial_{z} \neq 0$.

Eine Modellverteilung der magnetischen Feldstärke bei Annahme eines bestimmten Rotationsgesetzes ist nach einer Rechnung von A. Schlüter im Anhang wiedergegeben. Hierbei ist angenommen, daß die

5 a M. S chwarzschild, Astrophysic. J. 106, 427 [1947]. 


\begin{tabular}{|c|c|c|c|c|}
\hline \multicolumn{3}{|c|}{$\begin{array}{l}\text { Winkelgeschwindigkeit } \\
\text { () }\left[{ }^{0} / \mathrm{d}\right] \text { für }\end{array}$} & \multicolumn{2}{|c|}{$\begin{array}{c}\text { Magnetfeld } H \\
\text { für } \varphi=+45^{\circ} \text { in Gauß }\end{array}$} \\
\hline$\lambda$ & $\boldsymbol{r} / \boldsymbol{R}=0,2$ & $\boldsymbol{r} / R=0, \tilde{5}$ & $v / R=0,2$ & $\cdot R=0,5$ \\
\hline-3 & 1750 & 112 & -4220 & -1230 \\
\hline-2 & 350 & 56 & -452 & -352 \\
\hline-1 & 70 & $\approx 28$ & $-\quad 56$ & $-\quad 88$ \\
\hline $0^{*}$ & 14,0 & 14,0 & $0^{*}$ & $0^{*}$ \\
\hline+1 & 2,8 & 7,0 & 7,0 & $+\quad 29$ \\
\hline+2 & 0,56 & 3,5 & $+\quad 7,8$ & $+\quad 38$ \\
\hline
\end{tabular}

* Starre Rotation

Tab. 1. Winkelgeschwindigkeit und Magnetfeld bei nichtstarrer Rotation der Sonne.

$\left(\lambda=d \log \omega^{2} / d \log r ;\right.$ vgl. Anhang S. 70)

zentralen Teile der Sonne rascher rotieren als die äußeren. Der Verlauf der Feldlinien zeigt (qualitativ) Abb. 1.

Die Feldlinien der Magnetfelder, welche auf diese Art zustande kommen, verlaufen parallel zu Breitenkreisen unter der Oberfläche, und zwar bilden sie 2 antiparallele Bündel in den beiden Hemisphären; sie können daher nur durch lokale dynamische Störungen an die Oberfläche gebracht werden ${ }^{6}$. Hierbei muß der magnetische Druck $\mathfrak{S}^{2} / 8 \pi$ überwunden werden, der aber nur an der Oberfläche selbst dem Gasdruck oder dem Staudruck (für plausible Geschwindigkeitsbeträge) vergleichbar ist.

4. Es zeigt sich also, daß die durch Diffusionseffekte der beschriebenen Art entstehenden elektrischen Ströme bei durchaus bescheidenen Annahmen über die wirkenden potentiallosen Beschleunigungen bzw. die sie hervorrufenden Geschwindigkeiten ausreichen zur Erzeugung und Aufrechterhaltung von Magnetfeldern der auf Sternen beobachteten Größenordnung. Andererseits ergibt das spezielle Modell, das diskutiert wurde, nämlich rasche Rotation der zentralen Gebiete eines Sterns, noch keine geometrische Lage der Feldlinien, welche die Beobachtungen an den Sonnenflecken oder an den magnetischen Sternen verstehen läßt. Es ergibt sich also die Frage, ob sich andere plausible Modelle angeben lassen, welche hinsichtlich ihrer Gestalt den beobachteten Magnetfeldern besser entsprechen.

Hierzu gehen wir aus von der von Bjerknes für die Deutung der Sonnenflecken vorgeschlagenen Vorstellung und fragen, welches Rotationsgesetz $\omega(z, \bar{r})$ in der Hemisphäre auf zwei antiparallele geschlossene Ringe magnetischer Feldlinien führen würde, wie sie nach dem Bjerknesschen Modell der Sonnenaktivität

6 Vgl. T. G. C o w l ing, Monthly Notices Roy. astronom. Soc. 94, 39 [1934]. vorhanden sein müßten. Denken wir zunächst an ein Aktivitätsminimum, so sollte in einiger Tiefe unter der Oberfläche der eine Ring in etwa $30^{\circ}$ Breite und der andere dicht an der Äquatorebene stehen. Diese Ringe sind nach Bjerknes entgegengesetzt wirbelnde Wirbelringe. Zwischen ihnen wird also ständig etwas Materie aus dem Innern zur Oberfläche angesaugt (Abb. 2), oder von der Oberfläche ins Innere gepumpt. Im ersten Fall wird Materie mit retativ geringerem Rotationsmoment und relativ höherer Temperatur an die Oberfläche gebracht, so daß dort stationär ein relatives Minimum der Rotationsgeschwindigkeit und ein Maximum der Temperatur sein sollte, im zweiten Fall müßte es umgekehrt sein. Das Entgegengesetzte

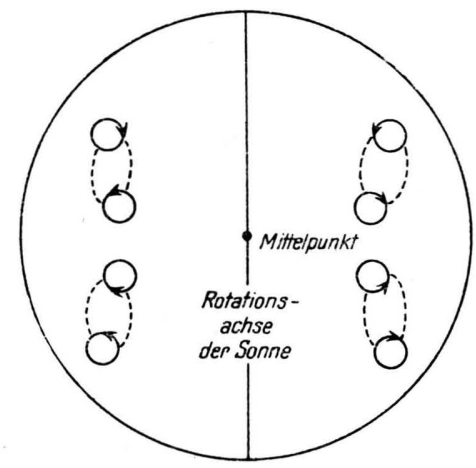

Abb. 2. Zur Kinematik der Sonnenflecken. Instantane Lage der Wirbelschläuche (nach Bjerknes) in einem Schnitt durch die Rotationsachse. Die postulierte langsame meridionale Zirkulation, welche die Wirbelschläuche in $2 \times 11$ Jahren umeinander herumführt, ist punktiert gezeichnet.

geschieht offenbar polarwärts des Wirbelrings in $30^{\circ}$ Breite und direkt am Äquator. Beide Effekte - die Änderung der Verteilung des Rotationsimpulses und der Temperatur - wirken im gleichen Sinn und müssen sich über die Bjerknessche Beziehung ins Gleichgewicht setzen. Die von Bjerknes postulierten Wirbelringe führen also gerade zu einer solchen Neigung der isobaren und der isothermen Flächen in der Umgebung der Wirbelringe, daß zwei antiparallele magnetische Ringe in jeder Hemisphäre resultieren. Bewegen sich die Wirbelschläuche im Verlauf des Zyklus umeinander, so kehren sich nach 11 Jahren auch die magnetischen Verhältnisse $\mathrm{um}^{7}$.

Die Dynamik und Genetik des postulierten Wirbelsystems sind hiermit natürlich keineswegs geklärt. Es sollte nur gezeigt werden, daß die von Bjerknes vorgeschlagene Kinematik der Sonnenflecken das Rota-

7 Hier kommen die Induktionseffekte ins Spiel, denen in den Arbeiten von A lf vé $\mathbf{n}^{1} \mathrm{u}$. Wa lé $\mathrm{n}$ die Hauptrolle zugewiesen wird. 
tionsgesetz bzw. die Druck-Dichteverteilung gerade in dem Sinne beeinflußt, wie es zur Erzeugung zweier antiparalleler magnetischer Ringe in jeder Hemisphäre erforderlich wäre. Die Zirkulation von 23 Jahren müßte offensichtlich auch die Magnetfelder herumführen. Damit die beobachteten Feldstärken von einigen 1000 Gauß erklärt werden, ohne daß zu hohe Geschwindigkeiten $v_{r}^{*}$ (d.h. zu große lokale Geschwindigkeitsunterschiede der Rotation um die Sonnenachse) postuliert werden müßten, sollten die Ringe größtenteils in sehr großer Tiefe $(\approx 100000 \mathrm{~km})$ verlaufen, wo die Leitfähigkeit hoch genug ist. Diese Annahme ist auch aus dem Grunde notwendig, weil sonst das an der Oberfläche beobachtete Rotationsgesetz deutlichere Spuren dieser Schwankungen im tieferen Innern zeigen müßte.

Es liegt nahe, die Frage aufzuwerfen, mit welcher Genauigkeit die Bjerknessche Beziehung gelten muß. Für die auftretenden Zirkulationsbeschleunigungen gilt

$$
\operatorname{rot} \dot{\mathfrak{v}}=\left\lceil\nabla \frac{1}{Q} \nabla P\right]
$$

Im Zustand stationärer Rotation muß dies gerade gleich rot $[\mathfrak{w}[\mathfrak{w r}]]$ sein, wo $[\mathfrak{w}[\mathfrak{w r}]]$ die Zentrifugalbeschleunigung ist. Wenn dies nicht der Fall ist, gibt die Beziehung die Beschleunigung der einsetzenden Zirkulationen. Die Größenordnung dieser Beschleunigung im Verhältnis zu $(1 / \varrho) \nabla P=g$ ist offenbar gegeben durch den Winkel der beiden Gradienten. Ein Winkel von z. B. $10^{-4,5}=6^{\prime \prime}$ führt wieder auf $\dot{\mathfrak{b}} \approx 1 \mathrm{~cm} / \mathrm{sec}^{2}$, d. h. in einem Tag auf Geschwindigkeiten der Ordnung $1 \mathrm{~km} / \mathrm{sec}$ und Wege der Ordnung $100000 \mathrm{~km}$, so daß in einigen Tagen jede Störung der Druckdichteverteilung der hier diskutierten Größenordnung auf einen Zustand führen muß, in dem die Bjerknessche Beziehung gilt.

Aus energetischen Gründen kann, sofern die Rotationsgeschwindigkeit nicht stark nach innen hin anwächst, das Spiel der Wirbelringe nur in einem schwach überdiabatisch geschichteten Gebiet stationär vor sich gehen. Falls die Wasserstoffzone die vom Verf. vorgeschlagene Tiefe besitzt ${ }^{s}$, würden in ihren tieferen Teilen vielleicht etwa die richtigen Verhältnisse vorkommen. Ein Überschuß über den adiabatischen Gradienten von $10^{-9} \% \mathrm{~cm}$ würde über Strecken

s L. B i e rm a n n, Astronom. Nachr. 264, 395 [1938]; Z. Astrophysik 21, 320 [1942].

9 Wirbelnde Beschleunigungsfelder erzeugen ebenfalls im Plasma elektrische Ströme, wie man aus Gl. (1) ableiten kann; vgl. hierzu A. S chlüter, Z. Naturforschg. 5 a, 72 [1950]. der Ordnung $10^{10} \mathrm{~cm}$ auf relative Temperaturunterschiede der Ordnung $10^{-5}$ führen; er erscheint dem vermuteten Verhältnis von $g^{*}: g$ angemessen.

Es zeigt sich also, daß man auf der Grundlage dieses Diffusionseffekts der freien Elektronen die Magnetfelder der Sonnenflecken wahrscheinlich verstehen wird, sobald man die Dynamik der Flecken verstanden hat.

Für die auf Sternen vom Typ 78 Virginis beobachteten Magnetfelder gibt vermutlich die Bjerknessche Vorstellung kein angemessenes Modell. Wir wollen daher eine andere Möglichkeit qualitativ diskutieren, welche durch die an den irdischen Drehstürmen beobachteten Verhältnisse nahegelegt wird. Bei diesen findet man außen (in großer Entfernung von der Achse) konstantes Rotationsmoment, innen konstante Winkelgeschwindigkeit (infolge Überwiegens der Reibung) und dazwischen ein Übergangsgebiet, in dem die angesaugte Materie offenbar Verzögerungen ausgesetzt ist, die eine wesentliche Komponente um die Achse herum (d. h. $\| \varphi)$ besitzen. Derartige Beschleunigungen führen daher im stationären Fall in einem Plasma auf axiale Magnetfelder ${ }^{9}$. Wir wollen versuchen, diese Vorstellung zu übertragen.

Hierzu betrachten wir einen rasch rotierenden Stern. $v_{r}{ }^{2}$ sei von der Ordnung $r g_{\mathrm{eff}} \approx k T / m$, der Stern sei also dicht an der Grenze der Rotationsinstabilität. In diesem Fall wird die Geschwindigkeit der meridionalen Zirkulationen kein allzu kleiner Bruchteil der Turbulenzgeschwindigkeit und der Rotationsgeschwindigkeit sein, und alle erwähnten $\mathrm{Ge}$ schwindigkeiten sind in grober Näherung von derselben Ordnung. Dafür stellen die adiabatischen Beziehungen keine gute Näherung mehr für das Innere dar, weil der turbulente Energietransport in diesen Sternen durch die Rotation sehr behindert wird. Die meridionale Zirkulation wird selbst, mindestens in großen Teilen des Sterninnern, turbulent $\operatorname{sein}^{10}$. Ein stationärer Zustand wird sich durch die Wechselwirkung von Zirkulation und turbulenter Reibung derart einstellen, daß trotz einer gewissen Zirkulation auch des Drehimpulses das Rotationsgesetz $\omega(z, r)$ sich nur säkular ändert.

Wenn diese Vorstellungen vom Aufbau und dem Rotationszustand der rasch rotierenden Sterne zutreffen, so müssen offenbar in bestimmten Zonen dieser Sterne stationär substantielle Beschleunigungs-

10 Allgemeine turbulenztheoretische Erwägungen deuten darauf hin, daß derartige Sterne Turbulenz viel größeren Maßstabes zeigen als langsam rotierende Sterne. Hrn. v. Weizs äcker bin ich für Bemerkungen zu diesem Punkt zu großem Dank verpflichtet. 
komponenten um die Rotationsachse herum auftreten, die, wie schon bemerkt, auf axiale Magnetfelder führen. Da die Zirkulationen das Magnetfeld (scheinbar) mitführen, ist die quantitative Diskussion sehr kompliziert, zumal die skizzierte Theorie des Aufbaus solcher Sterne ebenfalls erst wirklich durchgeführt werden müßte. Man kann mit den schon gegebenen Formeln nur abschätzen, daß magnetische Feldstärken der Ordnung $10^{3}$ bis $10^{4}$ Gauß mit Zirkulationsgeschwindigkeiten der Ordnung 1 bis $10 \mathrm{~km} / \mathrm{sec}$ erreicht würden. Diese erscheinen für rasch rotierende Sterne nicht unplausibel.

Zum Vergleich sei bemerkt, daß die Windgeschwindigkeiten in den irdischen Drehstürmen erhebliche Bruchteile der Schallgeschwindigkeit erreichen können.

5. In diesem Abschnitt diskutieren wir die Frage der Zeitskala ${ }^{11}$ und deuten die Ausdehnung dieser Betrachtungsweise auf den interstellaren Raum an. Die Einstelldauer eines Magnetfeldes, das durch eingeprägte elektrische Kräfte in ruhender Materie aufgebaut wird, und ebenso die Abklingzeit eines gegebenen Magnetfeldes (Energieverzehr durch Joulesche Wärme) beträgt größenordnungsmäßig $\sigma Q / c^{2}$, wo $Q$ der Querschnitt $\left[\mathrm{cm}^{2}\right]$ ist. Wenn nun aber durch Bewegungen der leitenden Materie elektrische Kräfte induziert werden, so kann hierdurch Bewegungsenergie in magnetische Energie übergehen. Wenn das Medium sehr gut leitet, so sind die Energieverluste durch Joulesche Wärme sehr klein, und die mechanische Energie wird quantitativ in magnetische Energie übergeführt. Die oben erwähnte Zeitskala ist dann irreführend. In diesem Fall bleibt der magnetische Fluß durch eine mit der Materie mitschwimmende geschlossene Linie für $\mathfrak{C}^{\star}=0$ in erster Näherung konstant. Der magnetische Fluß [Gauß $\times$ $\mathrm{cm}^{2}$ ] kann nur durch nicht-wirbelfreie und nichtmassenproportionale Kräfte geändert werden. Sobald man also damit zu rechnen hat, daß durch erzwungene Massenbewegungen und daraus resultierende Induktionseffekte die in Erscheinung tretenden magnetischen Feldstärken und Energiedichten erheblich vergrößert sind, muß man fragen, ob das Ringintegral der eingeprägten elektromotorischen Kräfte zur Erzeugung des beobachteten magnetischen Kraftflusses $\int \mathfrak{S}_{\mathrm{n}} d F\left[\mathrm{Gau} ß \mathrm{~cm}^{2}\right]$ hinreicht. Dies führt auf die Beziehung

$$
\begin{aligned}
-\frac{d}{d t} \int \mathfrak{S}_{n} d F=c \oint & \mathfrak{F}^{*} d_{\sigma}={ }^{c} \bar{m} \mathfrak{v}^{* 2} \\
& =10^{6}\left(\mathfrak{v}^{*} / \mathrm{km} / \mathrm{sec}\right)^{2} .
\end{aligned}
$$

${ }^{11}$ Vgl. hierzu T. G. Cow lin ${ }^{1}$.
Auf der Sonne beobachtet man in den Flecken Kraftflüsse von $10^{20}$ bis $10^{22} \mathrm{Gau} \mathrm{cm}^{2}$, auf den Sternen vom Typ 78 Virginis etwa $10^{25} \mathrm{Gau} \mathrm{cm}^{2}$. Nimmt man an, daß beide in $3 \cdot 10^{8}$ Jahren $\left(=10^{16} \mathrm{sec}\right)$ entstanden sind, so folgt für die Kraftflußänderung $10^{6}$ bzw. $10^{9} \mathrm{Gau} ß \mathrm{~cm}^{2} / \mathrm{sec}$, die Geschwindigkeiten $v_{r}{ }^{*}$ der Ordnung $1 \mathrm{~km} / \mathrm{sec}$ bzw. $30 \mathrm{~km} / \mathrm{sec}$ erfordern würden.

Die für das Sterninnere durchgeführte Behandlung läßt sich auf den interstellaren Raum übertragen; dabei sind die andersartigen Leitfähigkeitseigenschaften dieser hochverdünnten Materie (besonders in Gegenwart von Magnetfeldern) zu beachten ${ }^{12}$. Nimmt man auf $\left[10^{18} \mathrm{~cm}\right]^{2}$ einen Wirbelmechanismus an, der einen magnetischen Kraftfluß erzeugt, und rechnet mit $v_{r}=10 \mathrm{~km} / \mathrm{sec}$, so erhält man in der Zeit von $3 \cdot 10^{4}$ Jahren $\left(10^{12} \mathrm{sec}\right)$ einen Fluß von wieder $10^{20} \mathrm{Gau} \mathrm{cm}^{2}$, d. h. zunächst $10^{-16} \mathrm{Gauß}$. Ähnliche Feldstärken könnten aber auch durch von stark magnetischen Sternen abgegebene Magnetfelder erreicht werden. Durch Induktionseffekte und durch Entstehung immer neuer Turbulenzelemente könnte und sollte die magnetische Energiedichte bis in die Nähe der turbulenten Energiedichte erhöht werden. Diese Dinge sollen aber an anderer Stelle behandelt werden ${ }^{13}$.

An hang

$$
\text { M o de ll - Lös ung }
$$

Als vollständig integrierbares Modell für die Entstehung von Magnetfeldern durch nichtstarre Rotation betrachten wir einen Stern (Radius $R$ ), dessen sämtliche Volumenelemente um dieselbe Achse rotieren, und zwar mit einer Winkelgeschwindigkeit $\vec{\omega}$, die nur von dem Mittelpunktsabstand $r$ der betreffenden Stelle abhängt. Wir machen für $\vec{\omega}$ den speziellen Ansatz:

$$
\vec{c}=\mathfrak{a}\left(\begin{array}{l}
r \\
k
\end{array}\right)^{\lambda / 2} ; \quad \lambda>-5 .
$$

$\lambda$ ist hierbei ein willkürlicher Parameter, von dem nur gefordert werden muß, daß er größer als -5 ist, damit die Rotationsenergie endlich bleibt.

Wenn wir die Wirkungen des Strahlungsdrucks und der Thermodiffusion vernachlässigen, muß bei hydrostatischem Gleichgewicht der Druckgradient der Schwerebeschleunigung plus Zentrifugalbeschleunigung das Gleich-

${ }_{12} \mathrm{Zu}$ dieser Ausdehnung der Betrachtungen bin ich durch Diskussionen mit den HHrn. C. F. v. We i z$\mathrm{s}$ ä cker und $\mathrm{O}$. $\mathrm{H}$ axel angeregt worden. Die genauen Gleichungen, die das elektromagnetische Verhalteı solcher Materie in Gegenwart von äußeren Kräften beschreiben, werden von A. S chlüter, Z. Naturforschg. 5 a. 72 [1950], diskutiert.

${ }_{13}$ A. S c h l ü t e r u. L. B i e r m a n n, Interstellare Magnetfelder, Z. Naturforschg. 5 a [1950]. 
gewicht halten:

$$
\begin{aligned}
\frac{1}{N\left(m_{\mathrm{i}}+m_{\mathrm{e}}\right)} \nabla \mu_{\mathrm{G}} & =\mathfrak{g}+\left[\overrightarrow{\omega^{\prime}}[\vec{\omega} \mathrm{r}]\right] \\
& =\mathfrak{g}+\left(\frac{r}{k}\right)^{\hat{\lambda}}[\mathfrak{a}[\mathfrak{a} \mathfrak{r}]] .
\end{aligned}
$$

Hiermit wird das zu lösende Gleichungssystem nach (4), (6), (7)

$$
\begin{gathered}
\mathfrak{j}=\sigma\left(\mathfrak{E}+\mathfrak{E}^{\mathrm{e}}\right) ; \\
e \mathfrak{E}^{\mathrm{e}}=\frac{1}{2 N} \nabla p_{\mathrm{G}}=\bar{m} \mathfrak{g}+\bar{m}\left(\frac{r}{R}\right)^{j}[\mathfrak{a}[\mathfrak{a} \mathfrak{r}]] \\
\operatorname{div} \mathrm{j}=0 ; \quad \operatorname{rot}(\mathfrak{S}=0 .
\end{gathered}
$$

Wir machen nun die vereinfachende Annahme, daß die Leitfähigkeit $\sigma$ im Innern des Sterns konstant sei:

$$
\sigma=\begin{aligned}
& \sigma_{0} \text { für } r<R \\
& 0 \text { für } r>R
\end{aligned}
$$

Wir zerlegen nun $\mathfrak{E} \mathrm{e}_{\text {in }}$ den Wirbel- und den Potentialteil

$$
\mathfrak{C}^{\mathrm{e}}=\mathfrak{C H}^{*}+\mathfrak{C H}^{\prime} ; \quad \operatorname{div}\left(\mathfrak{C}^{*}=0 ; \quad \operatorname{rot} \check{C}^{\prime}=0,\right.
$$

also

$$
\operatorname{rot}\left(\mathfrak{5} *=\frac{\vec{m}}{e} \operatorname{rot}[\vec{\omega}[\vec{\omega} \mathfrak{x}]] ; \quad \operatorname{rot} \mathfrak{g}=0\right.
$$

Wie man durch Differenzieren verifizieren kann, ergibt sich die bis auf ein homogenes Glied eindeutige Lösung für $\mathfrak{F}^{*} \mathrm{zu}$ :

$$
\begin{array}{r}
\mathfrak{E}^{*}=\frac{m}{e} \frac{1}{\lambda+5}\left(\frac{r}{h}\right)^{\lambda}\{(\lambda+3) \mathfrak{a}(\mathfrak{a} \mathfrak{x}) \\
\left.-i \frac{\mathfrak{x}(\mathfrak{a} \mathfrak{r})^{2}}{r^{2}}-\mathfrak{a}^{2} \mathfrak{r}\right\} .
\end{array}
$$

Mit dem Ansatz $\tilde{\mathfrak{S}}=$ - $\mathfrak{E}^{\prime}$ könnten wir die Bedingung div $\mathrm{i}^{\prime}=0$ im Innern des Sterns befriedigen, jedoch nicht an der Oberfläche. Dort muß wegen dieser Bedingung verlangt werden, daß die Normalkomponente von $(\tilde{\mathfrak{E}}+(\tilde{y} e)$ verschwindet. Wir setzen daher an:

$$
\begin{aligned}
& \mathfrak{E}=\mathfrak{C}^{\prime \prime}-\mathfrak{E}^{\prime}, \text { d. h. } \mathfrak{E}^{+}+\mathfrak{E}^{\mathrm{e}}=\mathfrak{E}^{\prime \prime}+\mathfrak{C}^{*}, \\
& \operatorname{rot}\left(5 \prime \prime=0, \quad \operatorname{div}\left(\check{c}^{\prime \prime}=0 \text { für } r \leq R,\right.\right. \\
& \left(\mathfrak{r}\left(\check{c}^{\prime \prime}\right)+\left(\mathfrak{x}\left(\mathfrak{r}^{*}\right)=0 \text { für } r=R\right. \text {. }\right.
\end{aligned}
$$

Die einzige Lösung des Systems (25) mit dem Wert (24) für $\mathfrak{F}^{*}$ ist im Sterninnern

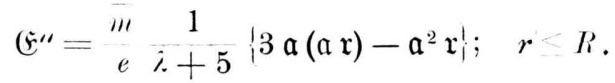

Damit folgt für die Stromdichte dort:

$$
\begin{aligned}
\mathrm{j}= & \sigma_{0}\left(\mathfrak{E}+\mathfrak{E}^{\mathrm{e}}\right)=\sigma_{0}\left(\mathfrak{F}^{\prime \prime}+\mathfrak{F}^{*}\right) \\
= & \frac{\sigma_{0} m}{e} \frac{1}{i+5}\left(1-\left(\frac{r}{R}\right)^{\hat{\lambda}}\right)\left\{\mathfrak{a} \mathfrak{a}(\mathfrak{a} \mathfrak{r})-\mathfrak{a}^{2} \mathfrak{r}^{!}\right. \\
& -\frac{\sigma_{0} m}{e} \frac{\lambda}{\lambda+5}\left(\frac{r}{R}\right)^{\lambda}\left\{\mathfrak{a}(\mathfrak{a} \mathfrak{r})-\frac{\mathfrak{r}(\mathfrak{a} \mathfrak{r})^{2}}{r^{2}}\right\} .
\end{aligned}
$$

Die magnetische Feldstärke ergibt sich als Lösung der Gleichungen

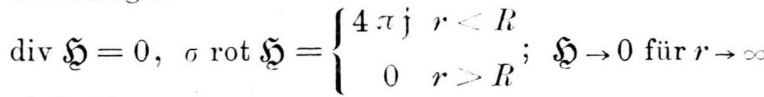
eindeutig $\mathrm{zu}$

$\mathfrak{S}=\left\{\begin{array}{cc}4 \pi \sigma_{0} \frac{m}{e c} \frac{\lambda+5}{\lambda}\left(1-\left(\frac{r}{R}\right)^{\hat{j}}\right)[\mathfrak{a} \mathfrak{r}](\mathfrak{a} \mathfrak{r}) & \text { für } r<R \\ 0 & \text { für } r \geq R,\end{array}\right.$

wie wieder durch Differenzieren zu bestätigen ist.

Die magnetischen Feldlinien verlaufen ganz im Innern als äquatorparallele Kreise um die Rotationsachse herum. $\mathrm{Da}$ sie daher mit den Stromlinien der Bewegung der Volumenelemente des Sternes zusammenfallen, ist nachträglich gerechtfertigt, daß wir die elektrodynamischen Wirkungen der Bewegung leitender Materie in einem Magnetfeld nicht berücksichtigt haben.

Zur Illustration berechnen wir die Feldstärken, die nach diesem Mechanismus auf der Sonne etwa entstehen können.

Wir setzen an

$e / \bar{m}=5,74 \cdot 10^{14}$ el. stat. cgs. (halbe Protonenmasse),

$\sigma=10^{17}$ el. stat. cgs. (richtig ungefähr für $r / R=0,4$ ),

$|\mathfrak{a}|=14^{\circ} / \mathrm{d}=2,83 \cdot 10^{-6} \mathrm{sec}^{-1}$ (Äquatorial-Winkel. geschwindigkeit der Sonne),

$R=R_{\odot}=6,95 \cdot 10^{10} \mathrm{~cm}$ (Sonnenradius).

Damit folgt für den Betrag $H$ des Magnetfeldes $(\varphi=$ heliographische Breite)

$$
H=1,41 \cdot 10^{3} \frac{(v / R)^{2}}{\hat{\gamma}+5}\left(1-\left(\frac{r}{R}\right)^{\hat{\lambda}}\right) \sin 2 \varphi[\text { Gau } \beta] .
$$

Die sich hieraus ergebenden Zahlwerte sind in der Tab. 1 (S. 68) angegeben. 